\title{
Correction: A tissue factor-cascade-targeted strategy to tumor vasculature: a combination of EGFP-EGF1 conjugation nanoparticles with photodynamic therapy
}

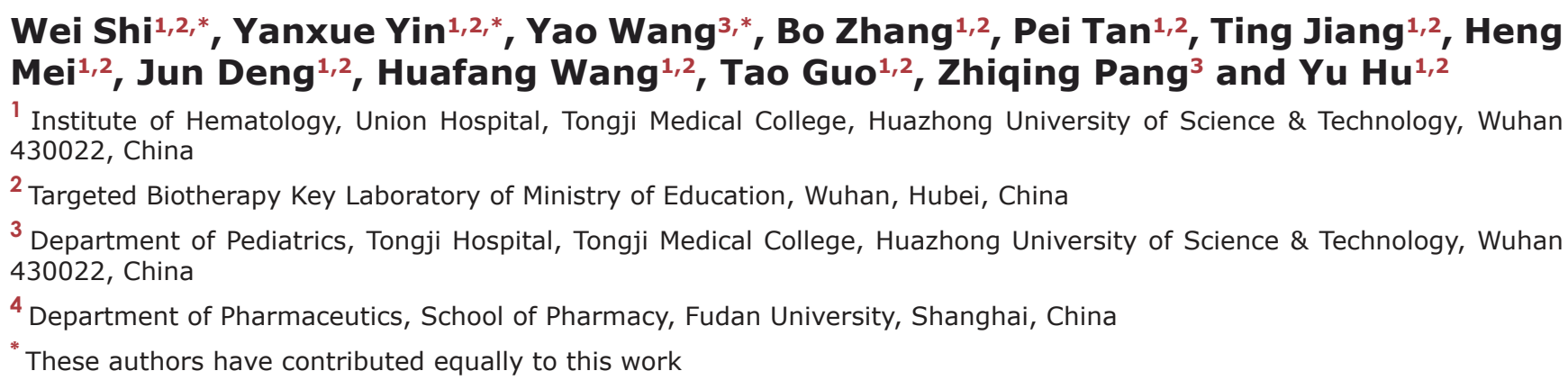

${ }^{1}$ Institute of Hematology, Union Hospital, Tongji Medical College, Huazhong University of Science \& Technology, Wuhan 430022, China

2 Targeted Biotherapy Key Laboratory of Ministry of Education, Wuhan, Hubei, China

${ }^{3}$ Department of Pediatrics, Tongji Hospital, Tongji Medical College, Huazhong University of Science \& Technology, Wuhan 430022, China

${ }^{4}$ Department of Pharmaceutics, School of Pharmacy, Fudan University, Shanghai, China

* These authors have contributed equally to this work

Published: April 03, 2018

Copyright: Shi et al. This is an open-access article distributed under the terms of the Creative Commons Attribution License 3.0 (CC BY 3.0), which permits unrestricted use, distribution, and reproduction in any medium, provided the original author and source are credited.

This article has been corrected: The proper name of the institution is as follows:

${ }^{1}$ Institute of Hematology, Union Hospital, Tongji Medical College, Huazhong University of Science \& Technology, Wuhan 430022, China

Original article: Oncotarget. 2017; 8:32212-32227. https://doi.org/10.18632/oncotarget.12922 